\title{
Addition of Gaussian random signals for peak to average power ratio reduction in OFDM systems
}

\begin{abstract}
This paper investigates the problem of peak-to-average power ratio (PAPR) in orthogonal frequency division multiplexing (OFDM) system. It presents a new PAPR reduction method based on addition of power of random signals in a complex Gaussian distribution form to the data constellation points in frequency domain. The added signals alter the constellation shape by shifting the constellation points from their original positions into new positions. This change in shape is accompanied by changes in the statistical properties and reduction in the PAPR value. There is no need to send side information to the receiver for signal recovery. This scheme significantly reduces the PAPR value without decrease in the bit rate or BER performance. Moreover, there is no out-of-band distortion resulted. For 64-PSK OFDM system using 128 data sub-carriers, $>4 \mathrm{~dB}$ reduction in the PAPR value is achieved by using the proposed PAPR reduction method.
\end{abstract}

